\title{
PRIMER REGISTRO DE MYLODON DARWINI OWEN (XENARTHRA, TARDIGRADA, MYLODONTIDAE) EN LA FORMACIÓN ARROYO FELICIANO (PLEISTOCENO TARDÍO), ENTRE RÍOS, ARGENTINA
}

\author{
EMMANUEL FAVOTTI, BRENDA S. FERRERO \& DIEGO BRANDONI \\ Laboratorio de Paleontología de Vertebrados, Centro de Investigaciones Científicas y Transferencia de Tecnología a la \\ Producción (CICYTTP), CONICET, E3105BWA Diamante, Entre Ríos, Argentina.efavotti@cicyttp.org.ar, \\ brendaferrero@cicyttp.org.ar,dbrandoni@cicyttp.org.ar
}

\begin{abstract}
FIRST RECORD OF MYLODON DARWINI OWEN (XENARTHRA, TARDIGRADA, MYLODONTIDAE) FROM ARROYO FELICIANO FORMATION (LATE PLEISTOCENE), ENTRE RIOS, ARGENTINA. In this contribution a new record of $M$. darwini Owen, based on an incomplete skull (MRHU-1) recovered from the Arroyo Feliciano Formation (late Pleistocene) on the Gualeguay River cliffs, nearly the city of Urdinarrain, is presented. Although the characteristic nasal arch of Mylodon darwini is not preserved at all, the morphology of the preserved portion and the presence of four maxillary lobated alveoli for the molariforms justify its assignment to this species. Based on its geographic and chronologic record, $M$. darwini has been associated to open environments with semiarid and cold climate (e.g. Pleistocene Pampean region and Patagonia of Argentina and Chile) as warm and humid climate (e.g. El Palmar Formation, late Pleistocene, Entre Ríos Province; late Pleistocene Arroyo Chuí, Brazil). The find of M. darwini in the Arroyo Feliciano Formation is important given that it corresponds to the first record of the species for the Formation and the second one for the province. Besides, the presence of M. darwini Arroyo Feliciano Formation corroborates the hypothesis that the genus inhabited in warm and humid climates.
\end{abstract}

Key words: Mylodontinae, Quaternary, South America, ground sloths, skull.

RESUMO - Neste trabalho damos a conhecer um novo registro de Mylodon darwini Owen, representado por um crânio incompleto (MRHU-1) encontrado nos sedimentos da Formação Arroyo Feliciano (Pleistoceno final) aflorante nas barrancas do rio Gualeguay, nas proximidades da localidade de Urdinarrain, Província de Entre Ríos, Argentina. Apesar do característico arco nasal de $M$. darwini não estar totalmente preservado no exemplar, a morfologia da porção preservada do rostro e a presença de quatro alvéolos superiores do tipo molariforme lobulado, justificam sua atribuição a esta espécie. Com base no seu registro, tanto geográfico quanto cronológico, $M$. darwini tem sido associado a ambientes abertos com climas semiáridos e frios (e.g. Pleistoceno da Região Pampeana e Patagônia da Argentina e do Chile) e a quentes e úmidos (e.g. Formação El Palmar, Pleistoceno final, Provincia de Entre Ríos; Pleistoceno superior de Arroio Chuí, Brasil). A presença de M. darwini na Formação Arroyo Feliciano é importante, pois corresponde ao primeiro registro da espécie para esta formação e o segundo para a província. Além disso, a presença de M. darwini na Formação Arroyo Feliciano corrobora com a hipótese de que o gênero habitava ambientes de clima quente e úmido.

Palavras-chave: Mylodontinae, Quaternário, América do Sul, preguiças, crânio.

\section{INTRODUCCIÓN}

La subfamilia Mylodontinae (Xenarthra, Tardigrada, Mylodontidae), tipificada por Mylodon Owen, 1839, es conocida y está documentada desde el Oligoceno tardío de Bolivia (Shockey \& Anaya, 2011), hasta el Holoceno de América del Sur y Pleistoceno tardío de América del Norte (McAfee, 2009; Brandoni et al., 2010). En Argentina, el clado es particularmente diverso durante el Mioceno tardío de la provincia de Entre Ríos, más precisamente en el miembro inferior de la Formación Ituzaingó (ver Brunetto et al., 2013), tradicionalmente conocido como "Mesopotamiense", unidad en la cual se ha registrado una alta diversidad de especies: Promylodon paranensis (Ameghino, 1883), Prolestodon paranensis (Ameghino, 1889), Prolestodon antiquus (Ameghino, 1885), Ranculcus scalabrinianus Ameghino,
1891, Strabosodon acuticavus Ameghino, 1891, S. obtusicavus Ameghino, 1891, Sphenotherus zavaletianus Ameghino, 1891 y Megabradys darwini Scillato-Yané, 1981 (Brandoni, 2013; Brandoni \& Favotti, 2013). Asimismo, durante el Cuaternario de esta provincia el clado Mylodontinae está representado por Mylodon darwini Owen, 1840 procedente de la Formación El Palmar, y por Glossotherium robustum (Owen, 1842) y Lestodon armatus Gervais, 1855 registrados en las formaciones Arroyo Feliciano y Salto Ander Egg (Ferrero, 2009, 2013; Brandoni et al., 2010; Brandoni \& Favotti, 2013; Brunetto et al., 2015). En lo que respecta a Mylodon, además del registro en Entre Ríos, este ha sido registrado en Argentina desde el Pleistoceno temprano hasta el Holoceno temprano en varias localidades de las provincias de Buenos Aires, Santa Fe, Córdoba y Santa Cruz (Scillato-Yané, 1976; Bargo \& Deschamps, 1996; Carlini \& Scillato-Yané, 1999; Cruz, 
2007; Brandoni et al., 2010; Borrero \& Martin, 2012; Martin, 2012). Además, para el resto de América del sur, Mylodon ha sido registrado en el Cuaternario de Bolivia (Marshall \& Sempere, 1991), Paraguay (Carlini \& Tonni, 2000), sur de Brasil (Oliveira, 1996; Ribeiro \& Scherer, 2009), Uruguay (Perea, 1998; Fariña et al., 2014) y Chile (Lopez Mendoza, 2007; Borrero \& Martin, 2012) (Figura 1A).

Desde un punto de vista geológico, el Pleistoceno tardío de la Provincia de Entre Ríos está representado por varias unidades portadoras de restos de vertebrados fósiles (e.g. formaciones El Palmar, Salto Ander Egg, Arroyo Feliciano y Tezanos Pinto). En el marco de recientes interpretaciones geológicas se destaca que la fauna del Pleistoceno tardío registrada en el sudoeste de la Provincia de Entre Ríos no proviene de la Formación Arroyo Feliciano como fue postulado (véase Tonni, 2004; Carlini et al., 2004; Noriega et al., 2004; Ferrero, 2009; Ferrero \& Noriega, 2009, entre otros), sino que proviene de la unidad geológica descripta como Formación Salto Ander Egg (Brunetto et al., 2009, 2015). Por lo tanto, el conocimiento del contenido de mamíferos fósiles de la Formación Arroyo Feliciano es escaso, y particularmente para la cuenca del río Gualeguay se restringe a unos pocos registros recientemente dados a conocer entre los que se reconocen ejemplares de: Toxodontidae, Megatheriidae, Mylodontidae,

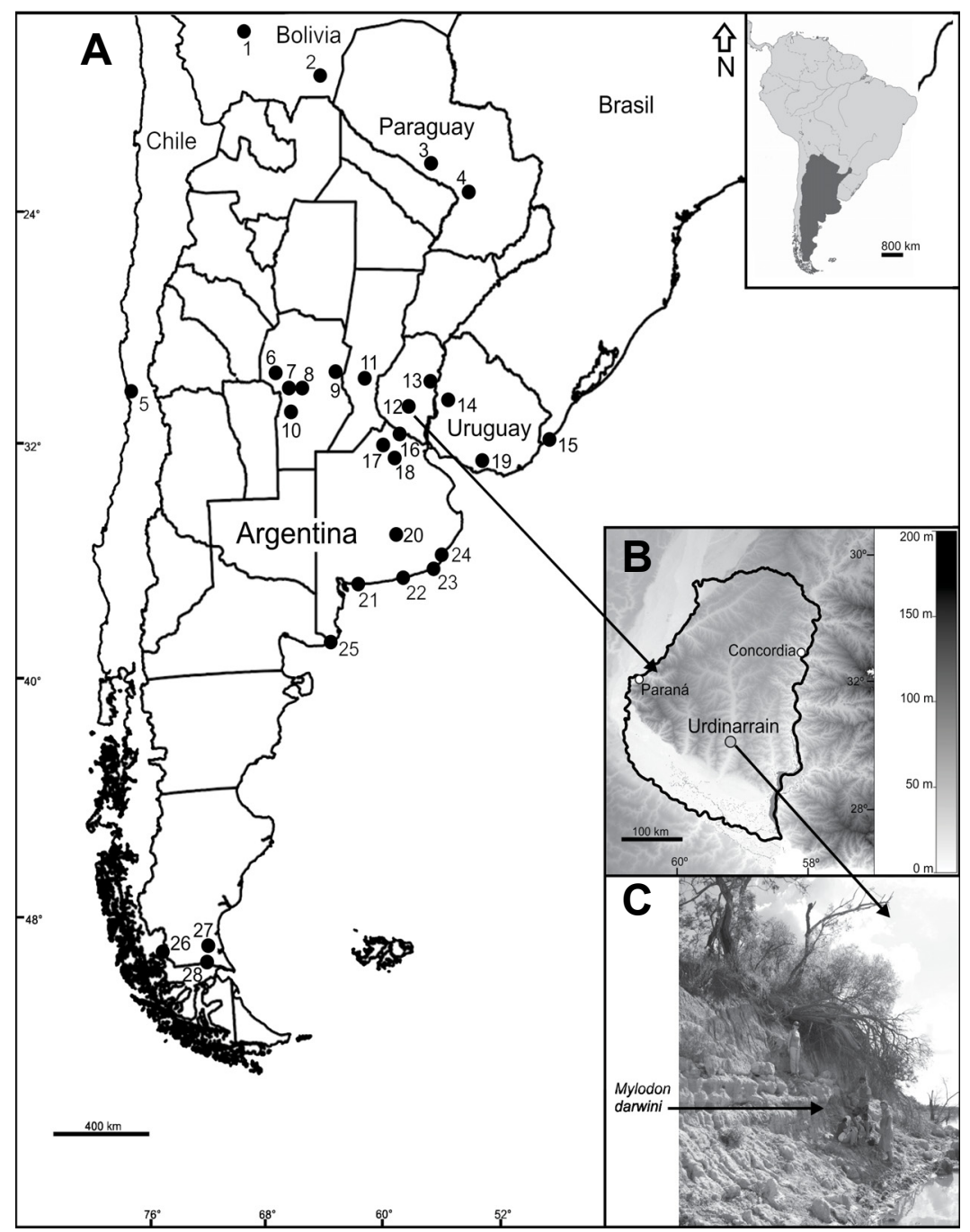

Figura 1. A, Mapa del sur de América del Sur mostrando la distribución de Mylodon darwini durante el Cuaternario; B, mapa de la Provincia de Entre Ríos indicando la ubicación geográfica de Urdinarrain; C, afloramientos de la Formación Arroyo Feliciano sobre el río Gualeguay. 1. Mojotorillo; 2, Nua Pua; 3, Riacho Negro; 4, General Bruguer; 5, Los Vilos; 6, San Alberto; 7, Calamuchita; 8, Río Tercero; 9, Santa María; 10, Río Cuarto; 11, Río Carcarañá; 12, Urdinarrain; 13, Concordia; 14, Departamento Río Negro; 15, Arroyo Chuí; 16, San Pedro; 17, Pergamino; 18. Salto; 19, Departamento de Canelones; 20, Olavarría; 21, Punta Alta; 22, Necochea; 23, Miramar; 24, Santa Clara; 25, Carmen de Patagones; 26. Cueva Ultima Esperanza; 27, Cueva Las Buitreras; 28, Cueva Fell, Cerro Sota, Pali Aike.

Figure 1. A, map of the southernmost part of South America showing the distribution of Mylodon darwini during the Quaternary; B, map of the Province of Entre Ríos indicating the geographic location of Urdinarrain; C, outcrops of the Arroyo Feliciano Formation on the Gualeguay River. 1, Mojotorillo; 2, Ñua Pua; 3, Negro Creek; 4, General Bruguer; 5, Los Vilos; 6, San Alberto; 7, Calamuchita; 8, Tercero River; 9, Santa María; 10. Cuarto River; 11, Carcarañá River; 12, Urdinarrain; 13, Concordia; 14, Río Negro Departament; 15, Chuí Stream; 16, San Pedro; 17, Pergamino; 18, Salto; 19, Canelones Departament; 20, Olavarría; 21, Punta Alta; 22, Necochea; 23, Miramar; 24, Santa Clara; 25, Carmen de Patagones; 26, Cueva Ultima Esperanza; 27, Las Buitreras Cave; 28, Fell Cave, Sota Cave, Pali Aike. 
Glyptodontidae, Pampatheriidae, Equidae, Cervidae, Camelidae, Tayassuidae y Gomphoteriidae (Brunetto et al., 2012; Ferrero, 2013; Ferrero \& Alcaraz, 2013; Ferrero et al., 2015). Además, fueron reportados ejemplares de Trachemys cf. T. dorbigni (Dúmeril \& Bibron, 1835) y Tapirus terretris Linnaeus, 1758, provenientes de sedimentos tentativamente asignados a la Formación Arroyo Feliciano reconocidos en el este de la Provincia de Entre Ríos (Tonni, 1992; de la Fuente et al., 2002).

En este trabajo se da a conocer un nuevo registro de Mylodon darwini representado por un cráneo incompleto recuperado de sedimentos de la Formación Arroyo Feliciano aflorante en las barrancas del río Gualeguay, en cercanías de la localidad de Urdinarrain, provincia de Entre Ríos (Figuras 1B,C).

Abreviaturas institucionales. MACNC Pv, Colección Paleontología Vertebrados del Museo de Antropología y Ciencias Naturales de Concordia, Concordia, Argentina; MACN Pv, Colección Paleontología Vertebrados del Museo Argentino de Ciencias Naturales "Bernardino Rivadavia", Buenos Aires, Argentina; MLP, Museo de La Plata, La Plata, Argentina; MMCIPAS, Museo Municipal Centro de Investigaciones Paleontológicas y Arqueológicas de Salto "José Fernando Bonaparte", Salto, Argentina; MNHN-BOL, Museo Nacional de Historia Natural, La Paz, Bolivia; MRHU, Museo Regional e Histórico de Urdinarrain, Urdinarrain, Entre Ríos, Argentina.

Abreviaturas anatómicas. M1-M4, molariformes superiores $1-4$.

\section{MATERIAL Y MÉTODOS}

El ejemplar aquí descripto se comparó con 11 ejemplares referidos a Mylodon darwini (Apéndice 1) y se consideraron cinco variables morfométricas craneanas (Figura 2): ABE, ancho bicondilar externo; ABI, ancho bicondilar interno; DCA, distancia desde el cóndilo hasta la parte posterior del alvéolo M4; LA, largo de la serie alveolar; LT, largo total.

\section{MARCO GEOLÓGICO}

La Formación Arroyo Feliciano (Pleistoceno tardío) está representada por facies sedimentarias de origen fluvial (Iriondo et al., 1985). La unidad presenta una facies compuesta por arenas finas pardo grisáceas con estratificación cruzada planar y otra facies fina de limos arenosos pardorojizos a gris con estructuras de laminación paralela horizontal y limos pardos poco estructurados, que varían de masivos a laminados groseramente. La primera puede interpretarse como una facies de mayor energía de flujo que representa el relleno de canal por migración de formas de lecho. La otra facies de materiales más finos constituye el relleno de la planicie de inundación (Brunetto et al., 2012). En el río Gualeguay constituye una terraza definida con un espesor típico de 2 a $5 \mathrm{~m}$ (Figura 1C). Desde el punto de vista paleontológico se registra hasta el momento unos pocos mamíferos (Ferrero, 2009, 2013; Ferrero \& Alcaraz, 2013; Ferrero et al., 2015) y restos de plantas fósiles (Moya et al., 2013; Moya \& Brea, 2015).

\section{PALEONTOLOGÍA SISTEMÁTICA}

Suborden TARDIGRADA Latham \& Davies, in Forster, 1795 Familia MYLODONTIDAE Gill, 1872

\section{Mylodon Owen, 1839}

Especie tipo. Mylodon darwini Owen, 1839.

Mylodon darwini Owen, 1839

(Figuras 3A-C)

Material referido. MRHU-1, cráneo incompleto que conserva parte de los nasales, frontales y parietales, anteriormente conserva parte del maxilar superior con los alvéolos pero sin los molariformes; además, se conserva el proceso postorbital derecho y la raíz maxilar del arco cigomático, en la región

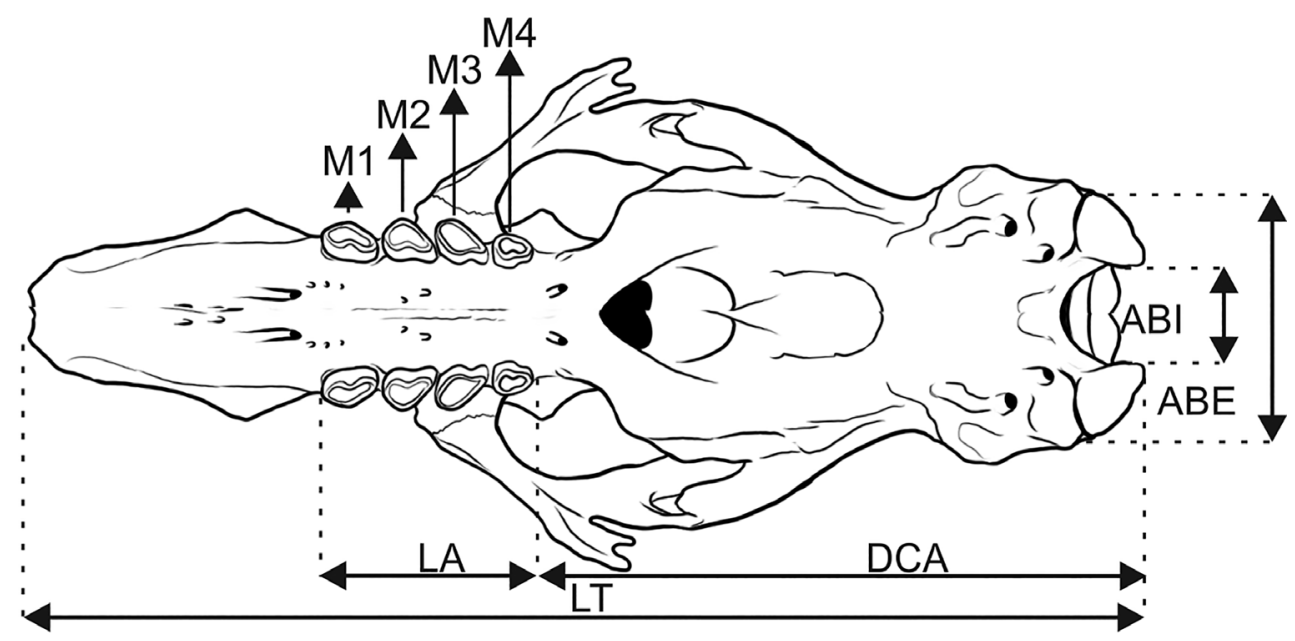

Figura 2. Medidas utilizadas en la comparación.

Figure 2. Measurements used for comparison. 
occipital se conservan ambos cóndilos, el foramen magnum y los forámenes condilares.

Procedencia geográfica. Barrancas del río Gualeguay en cercanías de la localidad de Urdinarrain (Departamento Gualeguaychú), Provincia de Entre Ríos, Argentina (32'42'10.79"'S, 597'7.61"O) (Figura 1B).

Procedencia estratigráfica. Facies sedimentarias de origen fluvial asignables a la Formación Arroyo Feliciano (Pleistoceno tardío) (Iriondo et al., 1985) (Figura 1C).

Descripción. El ejemplar MRHU-1 está representado por un cráneo incompleto al cual le falta, entre otros, la parte anterior del característico arco nasal. El cráneo es rectangular, puesto que en vista dorsal la distancia entre los márgenes laterales y la línea media es aproximadamente igual en toda su extensión (Figura 3A). Presenta un largo total conservado de $556 \mathrm{~mm}$. El techo del cráneo es ligeramente convexo. Los nasales se elevan gradualmente hacia la parte anterior constituyendo el punto más alto del cráneo, para luego descender hasta su borde anterior, lo que le confiere la convexidad característica (Figura 3B). En este ejemplar, al igual que en MACNC Pv 2334 y MACN Pv 13882, el nasal es poco elevado, en tanto que en MLP 3-122 es más notoria la elevación. La raíz maxilar del arco cigomático se implanta por encima de la serie alveolar (Figura 3B); su margen anterior se ubica sobre un plano cercano al borde anterior del M2, en tanto que su borde posterior se encuentra en un plano cercano al margen posterior al M3 (Figura 3C). En este aspecto, MRHU-1 difiere de otros ejemplares en los cuales la raíz maxilar se ubica más posterior; en MACNC Pv 2334 el margen anterior de la raíz maxilar se ubica cerca del plano que pasa por el borde posterior del M2 y su borde posterior se encuentra en un plano cercano al margen posterior al M4. Se conserva uno de los procesos postorbitales, éste está muy desarrollado, su base es amplia y su vértice es redondeado. En la parte posterior del cráneo, el foramen magnum es amplio $y$, al igual que en el ejemplar MLP 3-762, el margen ventral presenta forma de "V" (Figura 3C); en tanto que en MLP 3-764 y MACNC Pv 2334 el margen tiene forma de "U". Los cóndilos occipitales son hemielipsoidales $\mathrm{y}$ al igual que en los restantes milodontinos presenta un cuello corto y bien definido, siendo los cóndilos en Mylodon darwini más sésiles que en las especies de Glossotherium y Lestodon.

En lo que respecta a la dentición, la mayoría de las especies de Mylodontinae presentan cinco dientes superiores y cuatro inferiores (e.g. Glossotherium robustum, Lestodon armatus); Mylodon darwini constituye una excepción dado que se caracteriza por la pérdida del primer diente superior, por lo que la fórmula dentaria queda reducida a cuatro dientes superiores y cuatro inferiores ( $4 / 4$, todos de tipo molariforme). En MRHU-1, el largo total de la serie alveolar es de $121 \mathrm{~mm}$. En MRHU-1, la serie dentaria diverge levemente en línea recta hacia adelante (Figura 3C). El alvéolo del M1 es de contorno elíptico, con su diámetro mayor inclinado lateralmente con respecto al eje central del paladar. El alvéolo del M2 es subtriangular en sección; la cara lingual tiene una convexidad interna que insinúa dos lóbulos, en tanto que la cara labial es convexa. El alvéolo del M3 tiene un aspecto suboval, con su diámetro mayor inclinado lateralmente con respecto al eje central del paladar. El alvéolo del M4 es subtriangular en sección, con la pared lingual lobulada.

\section{DISCUSIÓN}

El cráneo de Mylodon darwini se caracteriza por la presencia de un arco nasal (formado por un proceso ascendente del premaxilar que se fusiona con una proyección descendente del nasal), la presencia de sólo cuatro molariformes de tipo lobulado en el maxilar y por presentar procesos postorbitales relativamente prominentes, como puede observarse en uno de los mejores ejemplares preservados (MACNC Pv 2334, Figuras 3D-F). Si bien en el ejemplar MRHU-1, el característico arco nasal no se ha preservado totalmente, ni tampoco los molariformes, la morfología de la porción preservada de rostro y la presencia de cuatro alvéolos superiores de tipo lobulado, justifican su asignación a M. darwini. Además, los valores de las medidas consideradas en el ejemplar MRHU-1 se encuentran dentro de los valores promedios calculados para la especie (Tabla 1).

La historia taxonómica de Mylodon y los géneros emparentados a los cuales se les ha referido especímenes de Mylodon (e.g. Glossotherium, Grypotherium Reinhardt, 1879, Neomylodon Ameghino, 1898) es compleja desde la propia y original descripción del género (ver Kraglievich, 1934). Tradicionalmente se ha considerado la existencia de cuatro especies de Mylodon: M. darwini, M. zeballozi Gervais \& Ameghino, 1880 M. listai Ameghino, 1898 y M. insigne Kraglievich , 1928 (ver Kraglievich, 1934; Esteban, 1996; Carlini \& Scillato-Yané, 1999), aunque la validez de algunas es dudosa (ver Kraglievich, 1934; Esteban, 1996; Brandoni et al., 2010). En este sentido, Esteban (1996) considera M. darwini como la única especie del género, siendo $M$. zeballozi, $M$. listai y $M$. insigne sinónimos juniors de esta, sin embargo esta sinonimia aún no ha sido formalmente establecida. Por su parte, Brandoni et al. (2010) indican que Mylodon darwini tendría una variabilidad individual mayor a la considerada en trabajos previos y sería la única especie (de las conocidas) correctamente diagnosticada y descripta; en tanto que Favotti (2015) propone sostener a $M$. insigne como una especie diferente de M. darwini. Si bien el número de materiales hallados y referidos a $M$. darwini, o a alguna de las otras especies del género, resultaría menor en comparación con aquellos ejemplares referidos a otros integrantes de la subfamilia (i.e. Glossotherium robustum, Lestodon armatus), el género presenta una amplia distribución geográfica, distribuyéndose desde el sur de Bolivia hasta el sur de la Patagonia argentina y chilena (Figura 1A).

Hasta la presente contribución, el registro de Mylodon darwini en la Provincia de Entre Ríos estaba acotado a la presencia en la Formación El Palmar en cercanías de la localidad de Concordia (Ferrero et al., 2007; Brandoni et al., 2010). Por lo tanto, el hallazgo de M. darwini en la Formación Arroyo Feliciano resulta significativo ya que corresponde al primer registro de la especie para la Formación y al segundo para la provincia.

Sobre la base de su registro tanto geográfico como cronológico, Mylodon darwini ha sido tradicionalmente 
A

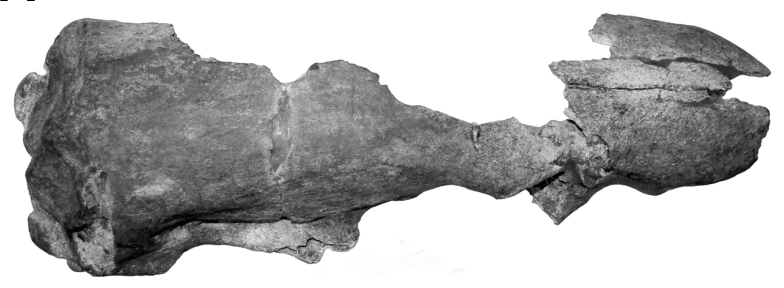

B

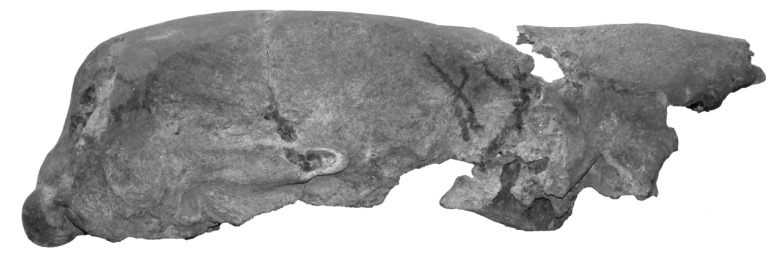

D

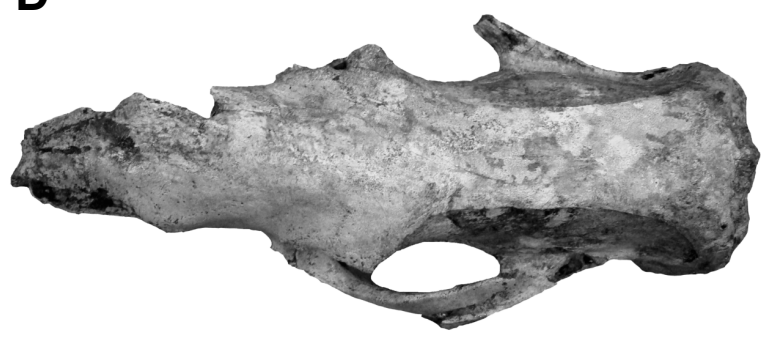

E
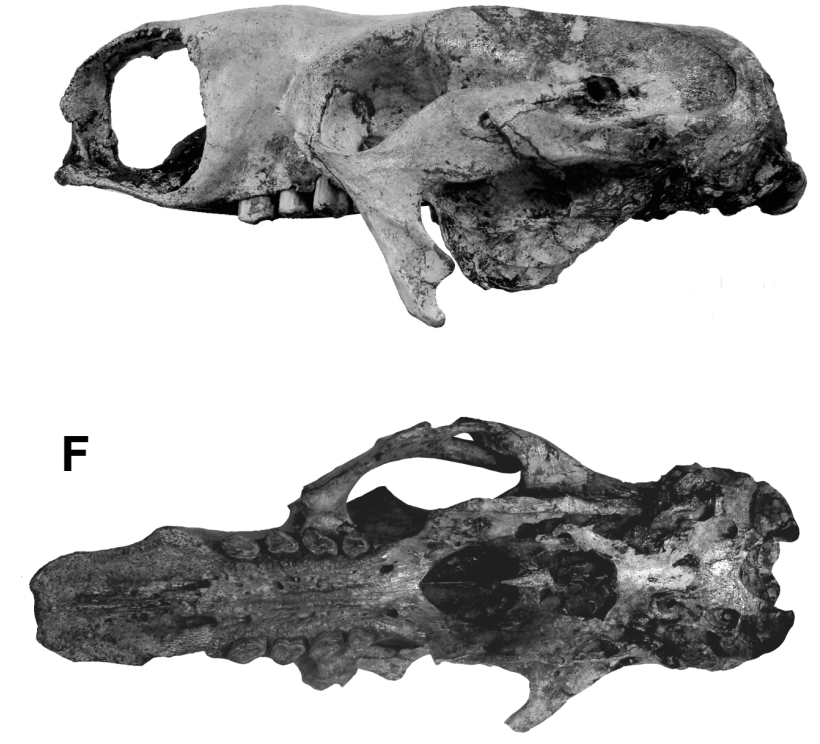

C

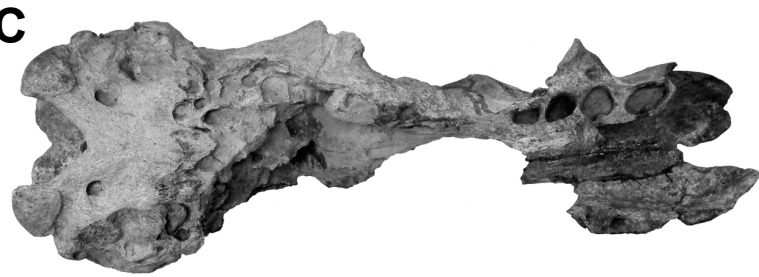

Figura 3. Cráneo de Mylodon darwini. A-C, MRHU-1; D-F, MACNC Pv 2334. A,D, vista dorsal; B,E, vista lateral; C,F, vista ventral. Escala $=100 \mathrm{~mm}$.

Figure 3. Skull of Mylodon darwini. A-C, MRHU-1; D-F, MACNC Pv 2334. A,D, dorsal view; B,E, lateral view; C,F, ventral view. Scale bar $=100 \mathrm{~mm}$.

Tabla 1. Medidas (mm) del cráneo de Mylodon darwini. Simbología: * Largo total conservado; \pm Desvío estándar.

Table 1. Measurements $(\mathrm{mm})$ of the skull of Mylodon darwini. Symbology: * Total preserved length; \pm Standard deviation.

\begin{tabular}{|c|c|c|c|c|c|}
\hline Ejemplar & LT & DCA & LA & $\mathrm{ABI}$ & $\mathrm{ABE}$ \\
\hline MRHU-1 & $556^{*}$ & 352 & 121 & 63 & 144 \\
\hline MACNC Pv 2334 & 605 & 335 & 109 & 51 & 139 \\
\hline MLP 3-122 & 660 & 365 & 133 & 56 & 150 \\
\hline MLP 3-762 & - & 385 & 123 & 56 & 160 \\
\hline MLP 3-763 & - & 330 & 126 & 58 & 139 \\
\hline MLP 3-764 & 715 & 365 & 126 & 60 & 177 \\
\hline MLP 36-VIII-12-1 & - & 383 & 122 & 50 & 148 \\
\hline MACN 15348 & - & - & 119 & 46 & 128 \\
\hline MACN Pv 13882 & - & - & 120 & - & - \\
\hline MMCIPAS B-50-2458 & - & 320 & 124 & 58 & 128 \\
\hline MNHN-BOL-V 006470 & 590 & 335 & 122 & 70 & 170 \\
\hline Rango & $590-715$ & $320-385$ & $109-133$ & $46-70$ & $128-177$ \\
\hline Promedio & $642,5 \pm 56,9$ & $352,2 \pm 25,27$ & $122,4 \pm 6,13$ & $56,1 \pm 6,9$ & $148,8 \pm 13,42$ \\
\hline
\end{tabular}


asociado a ambientes abiertos con climas semiáridos y fríos (Esteban, 1996; Tonni et al., 2003). Sin embargo, más recientemente, Brandoni et al. (2010) postularon que $M$. darwini presentaría una amplia tolerancia ecológica que le habría permitido habitar tanto en áreas con climas secos y fríos (e.g. Pleistoceno de la Región Pampeana y Patagonia) como en cálidos y húmedos (Pleistoceno tardío de Entre Ríos y Arroyo Chuí, Brasil). En este sentido, para la Formación El Palmar (Pleistoceno tardío de Entre Ríos) se ha propuesto la presencia de una comunidad vegetal compuesta por palmares asociados a gramíneas megatérmicas o mesotérmicas con la presencia de elementos arbóreos (e.g. Anacardiaceae, Mimosoideae, Fabacea, Lauraceae) y cuerpos de agua; dicha vegetación indicaría un clima tropical a subtropical húmedo para el momento del depósito de esta Formación (Zucol et al., 2005; Ramos, 2014; Ramos et al., 2012; Patterer et al., 2014). Respecto a la fauna registrada en la Formación El Palmar, se reconocen taxones típicos del Pleistoceno tardío de otras áreas de la Argentina, norte de Uruguay y sur de Brasil [e.g. Megatherium americanum Cuvier, 1796, Macrauchenia patachonica Owen, 1840, Stegomastodon platensis (Ameghino, 1888)], a los que se suma la presencia de Tapirus cf. T. terrestis, un taxón de distribución tropical y subtropical asociado a la presencia de cuerpos de agua (Ferrero, 2013; Ferrero et al., 2007). Para Arroyo Chuí, localizado en el extremo sur de Rio Grande do Sul en Brasil, se reconoce una importante fauna de mamíferos (e.g. Hydrochoerus Brisson, 1762, Myocastor Kerr, 1792, Tapirus Brisson, 1762, Tayassu Fischer, 1814, Catagonus Ameghino, 1904, Holmesina Simpson, 1930; ver Oliveira, 1996; Ribeiro \& Scherer, 2009; Pereira et al., 2012) indicativa tanto de áreas abiertas como de bosques subtropicales asociados a cuerpos de agua. No obstante, también se han encontrado roedores como Microcavia Gervais \& Ameghino 1880, Dolichotinae, Lagostomus cf. L. maximus (Desmarest, 1817) que sugieren ambientes áridos a semiáridos (ver Ubilla et al., 2007; Kerber et al., 2011) y grandes xenartros y ungulados nativos (Ribeiro \& Scherer, 2009; Scherer et al., 2009).

En lo que respecta a la Formación Arroyo Feliciano, Mylodon darwini se suma al elenco de mamíferos representados por taxones típicos del Pleistoceno tardío en la región [i.e., Toxodon platensis Owen, 1837, Megatherium americanum, Scelidodon Ameghino, 1881, Lestodon armatus, Glossotherium robustum, Glyptodon sp. Owen, 1838, Pampatherium Ameghino, 1891, Equus cf. (Amerhippus) neogeus Lund, 1840, Hippidion Owen, 1869, Hemiauchenia paradoxa H. Gervais \& Ameghino, 1880, Tayassu cf. T. pecari (Link, 1795), Hippocamelus sp. Leuckart, 1816 y Stegomastodon sp.] (Brunetto et al., 2012; Ferrero, 2013; Ferrero et al., 2015). Por su parte estudios geológicos (Iriondo et al., 1985) y paleobotánicos, que indican la presencia de especies de Styracaceae y Vochysiaceae (Moya \& Brea, 2015; Moya et al., 2013), proponen que el depósito de la Formación Arroyo Feliciano se produjo en condiciones climáticas más húmedas y cálidas que las actuales para la provincia, las cuales resultarían óptimas para la supervivencia de $M$. darwini en el área. Así, el registro de $M$. darwini en la Formación Arroyo Feliciano permite confirmar la idea de que Mylodon habitaría tanto en ambientes caracterizados por climas semiáridos y fríos, como en clima subtropical húmedo. Por otra parte, la presencia de un ejemplar de $M$. darwini, con un tamaño similar al de la media de la especie (Tabla 1) en un ambiente cálido y húmedo, no permitiría corroborar la hipótesis de Brandoni et al. (2010) quienes explican que el menor tamaño corporal del ejemplar procedente de la Formación El Palmar (i.e. MACNC Pv 2334, Figuras 3D-F) podría representar una respuesta a temperaturas ambientales más elevadas (i.e. regla de Bergmann).

\section{AGRADECIMIENTOS}

Agradecemos a S. Aizaga, M. Bidegain, T. Bourlot, S. Facello, J. Marognia, V. Micheli, F. Romero, R. Sanchez, R. Traverso, F. Zapata, D. Zapata y al personal de la Municipalidad y Museo de Urdinarrain: A. Espósito y N. Acosta por colaborar en la recuperación del ejemplar aquí descripto y en la protección del patrimonio paleontológico. A E. Brunetto por facilitar información sobre aspectos estratigráficos del área de estudio. Las revisiones de $\mathrm{A}$ Miño-Boilini, A.M. Ribeiro y un revisor anónimo mejoraron este manuscrito. Contribución a los proyectos PIP-Conicet 114-201101-00111, PIP-Conicet-112-201101-01024, PICT2013-0491-FONCyT-ANPCYT.

\section{REFERENCIAS}

Bargo, M.S. \& Deschamps, C.M. 1996. El registro de Mylodon Owen, 1840 (Mammalia, Tardigrada) en el Pleistoceno del sur de la Provincia de Buenos Aires. Comentarios sobre la distribución de los "sedimentos pampeanos". Ameghiniana, 33:343-348.

Borrero, L.A. \& Martin, F.M. 2012. Taphonomic observations on ground sloth bone and dung from Cueva del Milodón, Ultima Esperanza, Chile: 100 years of research history. Quaternary International, 278:3-11. doi:10.1016/j.quaint.2012.04.036

Brandoni, D. 2013. Los Tardigrada (Mammalia, Xenarthra) del Mioceno Tardío de Entre Ríos, Argentina. In: D. Brandoni \& J.I. Noriega (eds.) El Neógeno de la Mesopotamia argentina, Buenos Aires, Asociación Paleontológica Argentina, p. 135-144 (Publicación Especial 14).

Brandoni, D. \& Favotti, E. 2013. Los Mylodontidae (Mammalia, Xenarthra) del Cenozoico de Entre Ríos, Argentina: diversidad, distribución geográfica y cronológica. Ameghiniana, 50:R6.

Brandoni, D.; Ferrero, B.S. \& Brunetto, E. 2010. Mylodon darwini Owen (Xenarthra, Mylodontinae) from the Late Pleistocene of Mesopotamia, Argentina, whit remarks on individual variability, paleobiology, paleobiogeography, and paleoenvironment. Journal of Vertebrate Paleontology, 30:1547-1558. doi:10.10 80/02724634.2010.501449

Brunetto, E.; Ferrero, B.S.; Marino, J.; Tentor, F.; Sturtz, M.; Zamboni, L. \& Casanave, L. 2012. Evolución geológica del Pleistoceno del centro de Entre Ríos. In: CONGRESO ARGENTINO DE CUATERNARIO Y GEOMORFOLOGÍA, 5, 2012. Resúmenes, Río Cuarto, AACG, p. 132.

Brunetto, E.; Ferrero, B.S. \& Noriega, J.I. 2015. Late Pleistocene lithostratigraphy and sequences in the southwestern Mesopotamia (Argentina): evidences of the last interglacial stage. Journal of South American Earth Sciences, 58:111-128. doi:10.1016/j. jsames.2014.12.003 
Brunetto, E.; Ferrero, B.S.; Noriega, J.I.; Gottardi, M.G.; Zamboni, L. \& Gallina, G. 2009. Formación Salto Ander Egg: depósitos fluviales del pleistoceno superior, en el suroeste de Entre Ríos. In: CONGRESO DAASSOCIAÇAO BRASILEIRADE ESTUDOS DO QUATERNÁRIO, 12, 2009. Resúmenes, ABEQUA, La Plata, p. 260.

Brunetto, E.; Noriega, J.I. \& Brandoni, D. 2013. La Formación Ituzaingó en la provincia de Entre Ríos. Estratigrafía y edad. In: D. Brandoni \& J.I. Noriega (eds.) El Neógeno de la Mesopotamia argentina, Buenos Aires, Asociación Paleontológica Argentina, p. 13-27 (Publicación Especial 14)

Carlini, A.A. \& Scillato-Yané, G.J. 1999. Evolution of Quaternary Xenarthrans (Mammalia) of Argentina. Quaternary of South America and Antarctic Peninsula, 12:149-175.

Carlini, A.A. \& Tonni, E.P. 2000. Mamíferos fósiles del Paraguay. La Plata, Cooperación Técnica Paraguayo-Alemana, Imprenta Artes Gráficas San Miguel, 108 p.

Carlini, A.A.; Zurita, A.E.; Gasparini, G. \& Noriega, J.I. 2004. Los mamíferos del Pleistoceno de la Mesopotamia argentina y su relación con aquéllos del Centro-Norte de la Argentina, Paraguay y Sur de Bolivia, Sur de Brasil y Oeste de Uruguay: paleobiogeografía y paleoambientes. In: F.G. Aceñolaza (ed.) Temas de la Biodiversidad del Litoral fluvial argentino, Tucumán, Instituto Superior de Correlación Geológica, p. 83-90 (Miscelánea 12).

Cruz, L.E. 2007. Xenarthra (Mammalia) del Pleistoceno tardíoHoloceno temprano del Departamento Río Cuarto, provincia de Córdoba, Argentina. Aspectos bioestratigráficos. Ameghiniana, 44:751-757.

de la Fuente, M.; Noriega, J.I. \& Piña, C.I. 2002. Trachemys dorbigni (Duméril y Bibron, 1835) (Cryptodira: Emydidae) en el Pleistoceno tardío de la provincia de Entre Ríos, Argentina. Cuadernos de Herpetología, 16:65-72.

Esteban, G.I. 1996. Revisión de los Mylodontinae cuaternarios (Edentata-Tardigrada) de Argentina, Bolivia y Uruguay. Sistemática, filogenia, paleobiología, paleozoogeografía y paleoecología. Universidad Nacional de Tucumán, Facultad de Ciencias Naturales e Instituto Miguel Lillo, Tesis Doctoral, $335 \mathrm{p}$.

Fariña, R.A.; Tambusso, P.S.; Varela, L.; Czerwonogora, A.; Di Giacomo, M.; Musso, M.; Bracco, R. \& Gascue, A. 2014. Arroyo del Vizcaíno, Uruguay: a fossil-rich 30-ka-old megafaunal locality with cut-marked bones. Proceedings of the Royal Society of London B, 281:20132211. doi:10.1098/rspb.2013.2211

Favotti, E. 2015. Sobre el status taxonómico de Mylodon insigne Kraglievich (Xenarthra, Mylodontinae). Ameghiniana, 52:R16.

Ferrero, B.S. 2009. Diversidad y evolución de los Mamíferos del Cuaternario de la provincia de Entre Ríos Argentina. Aspectos bioestratigráficos y paleozoogeográficos de una fauna particular. Universidad Nacional de La Plata, Facultad de Ciencias naturales y Museo, Tesis Doctoral, $425 \mathrm{p}$.

Ferrero, B.S. 2013. Los vertebrados del Cuaternario de la provincia de Entre Ríos. Ameghiniana, 50:R11.

Ferrero, B.S. \& Alcaraz, M.A. 2013. Los ciervos (Artiodactyla, Cervidae) fósiles del Pleistoceno de la provincia de Entre Ríos, Argentina. Ameghiniana, 50:R47.

Ferrero, B.S.; Brandoni, D.; Noriega, J.I. \& Carlini, A.A. 2007. Mamíferos de la Formación El Palmar (Pleistoceno tardío) de la provincia de Entre Ríos, Argentina. Revista del Museo Argentino de Ciencias Naturales, 9:109-117.

Ferrero, B.S. \& Noriega, J.I. 2009. La paleontología de vertebrados en el Cuaternario de la provincia de Entre Ríos (Argentina): estado actual y perspectivas. In: A.M. Ribeiro; S.G. Bauermann \& C.S. Scherer (orgs.) Quaternario do Rio Grande do Sul: integrando conhecimentos, Porto Alegre, Sociedade Brasileira de Paleontologia, p. 207-215 (Monografias 1).

Ferrero, B.S.; Peralta, M.J.; Brunetto, E. \& Gasparini, G.M. 2015. Nuevos registros de mamíferos en la Formación Arroyo Feliciano (Pleistoceno tardío) en el tramo sur del río Gualeguay, provincia de Entre Ríos. Ameghiniana, 52:17.

Iriondo, M.; Ceruti, C. \& Tardivo, R. 1985. Geomorfología y cuaternario del tramo inferior del Arroyo Feliciano. Revista de la Asociación de Ciencias Naturales de Litoral, 16:149-156.

Kerber, L.; Lopes, R.P.; Vucetich, M.G.; Ribeiro, A.M. \& Pereira, J.C. 2011. Chinchillidae and Dolichotinae rodents (Rodentia, Hystricognathi, Caviomorpha) from the late Pleistocene of southern Brazil. Revista Brasileira de Paleontologia, 14:229238. doi: 10.4072/RBP.2011.3.03

Kraglievich, L. 1934. Contribución al conocimiento de Mylodon darwini Owen y especies afines. Revista del museo de la Plata, 34:255-292.

Lopez Mendoza, P. 2007. Tafonomía de los mamíferos extintos del Pleistoceno tardío de la costa meridional del semiárido de Chile (IV región - $32^{\circ}$ latitud S.). Alcances culturales y paleoecológicos. Revista de Antropología Chilena, 39:69-86. doi: $10.4067 / S 0717-73562007000100005$

Marshall, L.G. \& Sempere, T. 1991. The Eocene to Pleistocene vertebrates of Bolivia and their stratigraphic context: a review. Revista Técnica de Yacimientos Petroliferos Fiscales Bolivianos, 12:631-652.

Martin, F.M. 2012. Human-Carnivore Interaction at the End of the Pleistocene in Southern Patagonia, Chile. Journal of Taphonomy, 10:561-574.

McAfee, R.K. 2009. Reassessment of the cranial characters of Glossotherium and Paramylodon (Mammalia: Xenarthra: Mylodontidae). Zoological Journal of the Linnean Society, 155:885-903. doi:10.1111/j.1096-3642.2008.00468.x

Moya, E \& Brea, M. 2015. A new record of fossil Wood of Vochysiaceae from the Late Pleistocene (Arroyo Feliciano Formation), Argentina, South America. Revista Brasileira de Paleontologia, 18:83-90. doi:10.4072/rbp.2015.1.05

Moya, E.; Brea, M. \& Franco, M.J. 2013. Styracaceae del Pleistoceno superior (Formación Arroyo Feliciano) en el noreste de la Argentina. Ameghiniana, 50:R11.

Noriega, J.I.; Carlini, A.A. \& Tonni, E.P. 2004. Vertebrados del Pleistoceno tardío de la cuenca del Arroyo Ensenada (Departamento Diamante, Provincia de Entre Ríos). In: F.G. Aceñolaza (ed.) Temas de la Biodiversidad del Litoral fluvial argentino, Tucumán, Instituto Superior de Correlación Geológica, p. 71-76 (Miscelánea 12).

Oliveira, E.V. 1996. Mamíferos Xenarthra (Edentata) do Quaternário do Estado do Rio Grande do Sul, Brasil. Ameghiniana, 33:65-75.

Patterer, N.I.; Zucol, A.F. \& Brea, M. 2014. Análisis fitolíticos del perfil sedimentario Los Loros, de la Formación El Palmar (Pleistoceno superior), en el Parque Nacional El Palmar, Entre Ríos, Argentina. Acta Geológica Lilloana, 26:53-62.

Perea, D. 1998. Xenarthra fósiles del Uruguay: distribución estratigráfica caracterización osteológica y sistemática de algunos Tardigrada. PEDECIBA Biología, Universidad de la República, Tesis Doctoral, 107 p.

Pereira, J.C.; Lopes, R.P. \& Kerber, L. 2012. New Remains of late Pleistocene Mammals from The Chuí Creek, Southern Brazil. Revista Brasileira de Paleontologia, 15:228-239. doi:10.4072/ rbp.2012.2.10 
Ramos, R.S. 2014. Análisis paleoecológico preliminar de la flora de la Formación El Palmar (Pleistoceno Tardío), Entre Ríos, Argentina. Acta Geológica Lilloana, 26:63-74.

Ramos, R.S.; Brea, M. \& Kröhling D.M. 2012. Leños fósiles de la Formación El Palmar (Pleistoceno Tardío) en el Parque Nacional El Palmar, Entre Ríos, Argentina. Ameghiniana, 49:606-622. doi:10.5710/AMGH.17.5.2012.561

Ribeiro, A.M. \& Scherer, C.S. 2009. Mamíferos do Pleistoceno do Rio Grande do Sul, Brasil. In: A.M. Ribeiro; S.G. Bauermann \& C.S. Scherer (orgs.) Quaternario do Rio Grande do Sul: integrando conhecimientos, Porto Alegre, Sociedade Brasileira de Paleontologia, p. 171-192 (Monografias 1).

Scherer, C.S.; Pitana, V.G. \& Ribeiro, A.M. 2009. Proterotheriidae and Macraucheniidae (Litopterna, Mammalia) from the Pleistocene of Rio Grande Do Sul State, Brazil. Revista Brasilera de Paleontologia, 12:231-246. doi:10.4072/rbp.2009.3.06

Scillato-Yané, G.J. 1976. Sobre algunos restos de Mylodon (?) listai (Edentata, Tardigrada) procedentes de la Cueva "Las Buitreras" (Provincia de Santa Cruz, Argentina). Relaciones, 10:309-312.

Shockey, B.J. \& Anaya, F. 2011. Grazing in a new Late Oligocene mylodontid sloth and a mylodontid radiation as a component of the Eocene-Oligocene faunal turnover and the early spread of grasslands/savannas in South America. Journal of Mammalian
Evolution, 18:101-115. doi:10.1007/S10914-010-9147-5

Tonni, E.P. 1992. Tapirus Brisson, 1762 (Mammalia, Perissodactyla) en el Lujanense (Pleistoceno SuperiorHoloceno Inferior) de la Provincia de Entre Ríos, República Argentina. Ameghiniana, 29:3-8.

Tonni, E.P. 2004. Faunas y clima en el Cuaternario de la Mesopotamia argentina. In: F.G. Aceñolaza (ed.) Temas de la biodiversidad del litoral fluvial argentino, Tucumán, Instituto Superior de Correlación Geológica, p. 31-38 (Miscelánea 12).

Tonni, E.P.; Carlini, A.A.; Scillato-Yané, G.J. \& Figini, A.J. 2003. Cronología radiocarbónica y condiciones climáticas en la "Cueva del Milodón” (sur de Chile) durante el Pleistoceno Tardío. Ameghiniana, 40:609-615.

Ubilla, M.; Oliveira, E.V.; Rinderknecht, A. \& Pereira, J.C. 2007. First record of Microcavia (Rodentia, Caviidae) in Brasil (late Pleistocene, Rio Grande do Sul). Ameghiniana, 44:R42.

Zucol, A.F.; Brea, M. \& Scopel, A. 2005. First record of fossil wood and phytolith assemblages of the Late Pleistocene in El Palmar National Park (Argentina). Journal of South American Earth Sciences, 20:33-43. doi:10.1016/j.jsames.2005.07.007

Received in December, 2014; accepted in July, 2015.

Apéndice 1. Ejemplares utilizados en este estudio.

Appendix 1. Specimens used in this study.

MRHU-1, cráneo incompleto, procedente de la Formación Arroyo Feliciano (Pleistoceno tardío), Urdinarrain, Provincia de Entre Ríos, Argentina.

MACNC Pv 2334, cráneo casi completo y mandíbula, procedente de la Formación El Palmar (Pleistoceno tardío), Concordia, Provincia de Entre Ríos, Argentina. MLP 3-122, cráneo incompleto, procedente del Pleistoceno tardío de la Provincia de Buenos Aires, Argentina.

MLP 3-762, cráneo incompleto, procedente del Pleistoceno tardío de la localidad de Olavarría, Provincia de Buenos Aires, Argentina.

MLP 3-763, cráneo incompleto, procedente del Pleistoceno de la Provincia de Buenos Aires, Argentina.

MLP 3-764, cráneo casi completo, procedente del Pleistoceno de la Provincia de Buenos Aires, Argentina.

MLP 36-VIII-12-1, cráneo incompleto, procedente del Pleistoceno de Estación Bunge, Provincia de Buenos Aires, Argentina.

MACN 15348, cráneo incompleto, en parte reconstruido, procedente del Pleistoceno de la Provincia de Buenos Aires, Argentina.

MACN Pv 13882, cráneo incompleto, procedente del Pleistoceno de Necochea, Provincia de Buenos Aires, Argentina.

MMCIPAS B-50-2458, cráneo incompleto, procedente del Pleistoceno de la localidad de Salto, Provincia de Buenos Aires, Argentina.

MNHN-BOL-V 006470, cráneo casi completo, procedente del Pleistoceno de Mojotorillo, Departamento Potosí, Bolivia. 